\title{
The Research on the Production of Bio-based Polyamides 56 Sheath-core Composite Modified Fiber
}

\author{
Shouyun Zhang, Jinghong Ma* \\ State Key Laboratory for Modification of Chemical Fibers and Polymer Materials, College of Materials Science and Engineering, Donghua \\ University, Shanghai, P. R. China
}

Email address:

Syzhang2008741208@163.com (Shouyun Zhang),mjh68@dhu.edu.cn (Jinghong Ma)

*Corresponding author

To cite this article:

Shouyun Zhang, Jinghong Ma. The Research on the Production of Bio-based Polyamides 56 Sheath-core Composite Modified Fiber. International Journal of Materials Science and Applications. Vol. 5, No. 2, 2016, pp. 79-83. doi: 10.11648/j.ijmsa.20160502.17

Received: February 28, 2016; Accepted: April 25, 2016; Published: May 4, 2016

\begin{abstract}
In order to improve the moisture absorption, the antistatic properties of chemical fiber and improve the wearing comfort of fabric, the author has studied the modification and the production process of the sheath-core composite fiber which made of bio-based polyamide 56 and PET. Experiments show: selecting the appropriate production technology, using the bio-based polyamide 56 as the cortical structures, using the pet as the core structure we can produce high quality Sheath-core composite modified fiber by Composite spinneret. The Fiber has good moisture absorption performance and easy to be dyed. The fabric feel satiny soft and has a good elasticity, antistatic effect. It wears comfortable and the conformal performance is good.
\end{abstract}

Keywords: Bio-based, Polyamide 56, Sheath-corecomposite, Hygroscopicity, Antistatic Property

\section{Introduction}

The conventional product capacity of Polyester has been serious excess demanding. Its profit falling dramatically. The whole industry is in the loss. However, because of the feel of pet fiber is stiff and smooth and its wax feeling is strong, poor hygroscopicity and poor Antistatic performance, the wearing comfort and feel of fabric is very poor, this restricts the development of polyester fiber application to a great extent. Bio-based polyamides 56 production cost is low. Its macromolecular odd-even unit structure can form a large number of unsaturated hydrogen bond. It make the fiber and fabric has good flexibility, strong hygroscopicity, dyeing rate higher and Its production processing performance and process is close to PET. It suit to produce the skin-core structure composites fiber with PET and its compatibility with PET is better than polyamide 6 and polyamide 66 . It can improve the combination of skin-core structure interface and avoid the relative slip during the Sheath layer and core layer structure and keep the fiber surface undamaged when it was been drawn in the after processing so we can improve the performance of sheath-core composite fiber. The rigidity of PET is relatively large. As a textile material it has a good effect on drapability and conformity. Bio-based polyamides 56 has a good hygroscopicity. We can use the bio-based polyamides 56 as sheath and use PET as the core layer, adding a certain proportion fluorescent whitening agent to the Bio-based polyamides 56, using sheath-core composite spinneret structure components, we had developed and produced Bio-based polyamides 56 sheath-core composite modified fiber. The fabric has good soft feel, quite rich conformity and Wearing comfort. The author had analyzed the compound proportion, production process, product, moisture absorption and antistatic properties of $150 \mathrm{D} / 72 \mathrm{~F}$ bio-based polyamides 56 sheath-core composite filament fiber DTY and FDY.

\section{Experimental Section}

\subsection{Materials}

Bio-based polyamides 56 chip: the United States DuPont Co., Ltd. fiber level.

Pet chip: Zhejiang Hengyi Polymer Co., Ltd, fiber level.

The major physical performance indicators of PA56 shown in table 1.

The major physical performance indicators of pet shown in table 2 . 
Table 1. The major physical performance indicators of PA56.

\begin{tabular}{ll}
\hline Item & indicators \\
\hline Inherent viscosity $/\left(\mathrm{dl} . \mathrm{g}^{-1}\right)$ & 1.350 \\
Melting point $/{ }^{\circ} \mathrm{C}$ & 256 \\
$\mathrm{w}(\mathrm{H} 2 \mathrm{O}) / \%$ & 0.60 \\
The amino content $/ \%$ & 0.042 \\
End carboxyl content $/ \%$ & 0.050 \\
Color value $\mathrm{L}$ & 70 \\
Color value $\mathrm{b}$ & 16.2 \\
The yellow index & 32.2 \\
\hline
\end{tabular}

Table 2. The major physical performance indicators of PET.

\begin{tabular}{ll}
\hline Item & indicators \\
\hline Inherent viscosity $/\left(\mathrm{dl} . \mathrm{g}^{-1}\right)$ & 0.643 \\
Melting point $/{ }^{\circ} \mathrm{C}$ & 251 \\
The amino content $/ \%$ & 17 \\
Color value $\mathrm{b}$ & 3.2 \\
Color value $\mathrm{L}$ & 94 \\
$\mathrm{w}(\mathrm{DEG}) / \%$ & 0.40 \\
$\mathrm{w}\left(\mathrm{TiO}_{2}\right) / \%$ & 0.21 \\
\hline
\end{tabular}

\begin{tabular}{ll}
\hline Item & indicators \\
\hline $\mathrm{w}(\mathrm{ash}) / \%$ & 0.23 \\
$\mathrm{w}(\mathrm{Fe}) / 10^{-6}$ & 4.0 \\
$\mathrm{w}\left(\mathrm{H}_{2} \mathrm{O}\right) / \%$ & 0.40 \\
\hline
\end{tabular}

\subsection{Main Equipments}

Beijing De Hou Pu Chemical Technology Co., Ltd manufacture DGD67-1 type fluidized early crystallization and filling tower for drying equipment;

Beijing Zhong Li Machine Chemical Fiber Engineering Technology Co.,

Ltd JK81 - 105 - x 25-00 length to diameter ratio of 25:1 screw extruder;

Beijing Zhong Li FDY/POY composite spinning and winding machine;

German Bamarg Co., Ltd, ATT type false twist texturing machine;

\subsection{The Major Technological Process}

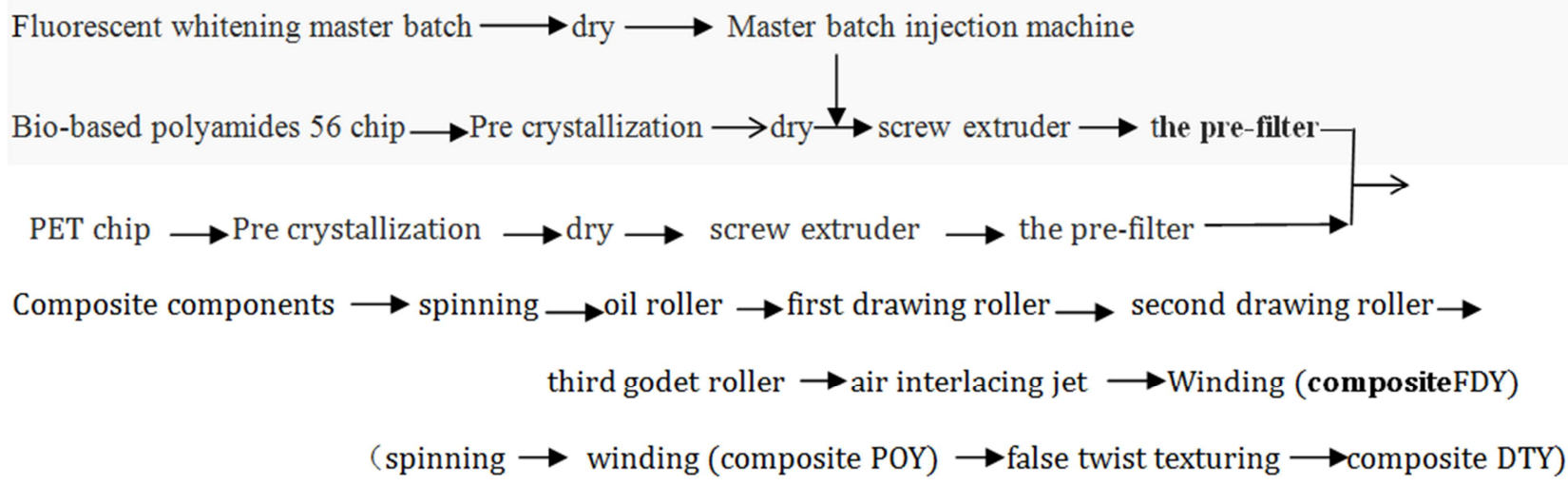

\subsection{The Production Process}

The main spinning process parameters are shown in table 3

Table 3. The spinning process parameters of Bio-based polyamides 56 Sheath-core composite modified FDY fiber.

\begin{tabular}{llll}
\hline Item & parameter & Item & parameter \\
\hline PET pre-crystallization temperature $/{ }^{\circ} \mathrm{C}$ & 160 & PA56 pre-crystallization temperature $/{ }^{\circ} \mathrm{C}$ & 140 \\
PET drying temperature $/{ }^{\circ} \mathrm{C}$ & 153 & PA56 drying temperature $/{ }^{\circ} \mathrm{C}$ & 135 \\
Master batch drying temperature $/{ }^{\circ} \mathrm{C}$ & 120 & Master batch drying time $/ \mathrm{h}$ & 6 \\
PET spinning temperature $/{ }^{\circ} \mathrm{C}$ & 293 & PA56 spinning temperature $/{ }^{\circ} \mathrm{C}$ & 285 \\
PET Pre-crystallization time $/ \mathrm{min}$ & 35 & Oil pump frequency $/ \mathrm{Hz}$ & 36 \\
PA56 pre-crystallization time $/ \mathrm{min}$ & 30 & Side wind temperature $/{ }^{\circ} \mathrm{C}$ & 25 \\
PET drying time $/ \mathrm{h}$ & 10 & Side wind Speed $/\left(\mathrm{m} . \mathrm{s}^{-1}\right)$ & 0.45 \\
PA56 drying time $/ \mathrm{h}$ & 30 & Side wind humidity $/ \%$ & 85 \\
PA56 After filter pressure $/ \mathrm{Mpa}$ & 93 & PA56 and PET composite proportion & $70: 30$ \\
PET After filter pressure $/ \mathrm{Mpa}$ & 92 & spinding initial pressure $/ \mathrm{Mpa}$ & 16.8 \\
1 draw roller temperature $/{ }^{\circ} \mathrm{C}$ & 80 & 2 draw roller temperature $/{ }^{\circ} \mathrm{C}$ & 125 \\
Pre-interlacing pressure $/ \mathrm{Mpa}$ & 0.08 & nterlacing pressure $/ \mathrm{Mpa}$ & 0.25 \\
Winding Angle $/\left({ }^{\circ}\right)$ & 5.2 & Draw ratio & 2.6 \\
\hline
\end{tabular}

The temperature of each zone of screw are shown in table 4 .

Table 4. The temperature of each zone of screw.

\begin{tabular}{llllll}
\hline Each zone of screw & first & second & third & fourth & fifth \\
\hline PET screw temperature $\left({ }^{\circ} \mathrm{C}\right)$ & 282 & 286 & 288 & 290 & 292 \\
PA56 screw temperature $\left({ }^{\circ} \mathrm{C}\right)$ & 270 & 274 & 276 & 279 & 283 \\
\hline
\end{tabular}


The Main parameters of false texturing process are shown in table 5 .

Table 5. The Main false texturing process parameters of Bio-based polyamides 56 Sheath-core composite modified fiber.

\begin{tabular}{llll}
\hline Item & parameter & Item & parameter \\
\hline Yarn speed $/ \mathrm{m} / \mathrm{min}$ & 680 & $\mathrm{D} / \mathrm{Y}$ & 1.550 \\
Draw Ratio & 1.68 & $\mathrm{OF} 2 / \%$ & 5.85 \\
$\mathrm{OF} 3 / \%$ & 4.87 & $\mathrm{H} 1 /{ }^{\circ} \mathrm{C}$ & 190 \\
$\mathrm{H} 2 /{ }^{\circ} \mathrm{C}$ & $\mathrm{OFF}$ & Winding Angle $/{ }^{\circ}$ & 14.8 \\
\hline
\end{tabular}

\subsection{The Finished Product Performance Indicators}

Table 6. The finished product performance indicators of Bio-based polyamides 56 Sheath-core composite POY fiber.

\begin{tabular}{llll}
\hline Item & parameter & Item & parameter \\
\hline density/dtex & 250.1 & elongation $/ \%$ & 125.3 \\
Density $\mathrm{CV} / \%$ & 1.3 & elongation $\mathrm{CV} / \%$ & 2.6 \\
intensity $/ \mathrm{CN} . \mathrm{dtex}^{-1}$ & 2.5 & oiling $/ \%$ & 0.60 \\
Intensity $\mathrm{CV} / \%$ & 1.0 & fiber value $\mathrm{cv} / \%$ & 1.2 \\
\hline
\end{tabular}

The finished product performance indicators of $162 \mathrm{dtex} / 72 \mathrm{f}$ Bio-based polyamides 56 Sheath-core composite FDY fiber are shown in table 7.

Table 7. The performance indicators of Bio-based polyamides 56 Sheath-core composite FDY fiber.

\begin{tabular}{llll}
\hline Item & parameter & Item & parameter \\
\hline density/dtex & 162.2 & elongation/\% & 32.3 \\
Density CV/\% & 1.3 & elongationCV/\% & 2.6 \\
intensity/CN.dtex ${ }^{-1}$ & 4.6 & oiling & 1.1 \\
$\begin{array}{l}\text { Intensity CV/\% } \\
\text { Boiling water }\end{array}$ & 1.7 & fiber value cv/\% & 1.6 \\
shrinkage /\% & 7.8 & $\begin{array}{l}\text { Dry heat } \\
\text { shrinkage rate/\% }\end{array}$ & 9.2 \\
$\begin{array}{l}10 \% \text { Elastic } \\
\text { response rates/\% }\end{array}$ & $99.3 \%$ & $\begin{array}{l}\text { 20\% Elastic } \\
\text { response rates } / \%\end{array}$ & $99.5 \%$ \\
\hline
\end{tabular}

The finished product performance indicators of $162 \mathrm{dtex} / 72 \mathrm{f}$ Bio-based polyamides 56 Sheath-core composite DTY fiber are shown in table 8

Table 8. The performance indicators of Bio-based polyamides 56 Sheath-core composite DTY fiber.

\begin{tabular}{llll}
\hline Item & indicators & Item & indicators \\
\hline density/dtex & 162.4 & elongation/\% & 28.8 \\
Density CV/\% & 2.1 & elongation CV/\% & 2.1 \\
intensity/CN.dtex ${ }^{-1}$ & 4.5 & oiling/\% & 2.5 \\
$\begin{array}{l}\text { Intensity CV/\% } \\
\begin{array}{l}10 \% \text { Elastic response } \\
\text { rates/\% }\end{array}\end{array}$ & 1.5 & fiber value cv/\% & 1.45 \\
\hline
\end{tabular}

The composite performance indicators are shown in table 9

Table 9. The composite performance indicators of of Bio-based polyamides 56 Sheath-core composite fiber.

\begin{tabular}{l|l|l}
\hline Indicators & FDY & DTY \\
\hline Fiber moisture regain/\% & 2.6 & 2.9 \\
\hline $\begin{array}{l}\text { Fabric moisture absorption level/mm } \\
\text { resistance } / \Omega \cdot \mathrm{cm} \text { (temperature: } 20^{\circ} \mathrm{C},\end{array}$ & 280 & 300 \\
$\begin{array}{l}\text { humidity: } 65 \% \text { ) } \\
\text { Color value L }\end{array}$ & $2.0 \times 10^{6}$ & $2.2 \times 10^{6}$ \\
Color value b & 92 & 91.5 \\
The yellow index (YI) & 2.7 & 2.8 \\
\hline
\end{tabular}

\section{Results and Discussion}

\subsection{Fiber Structure}

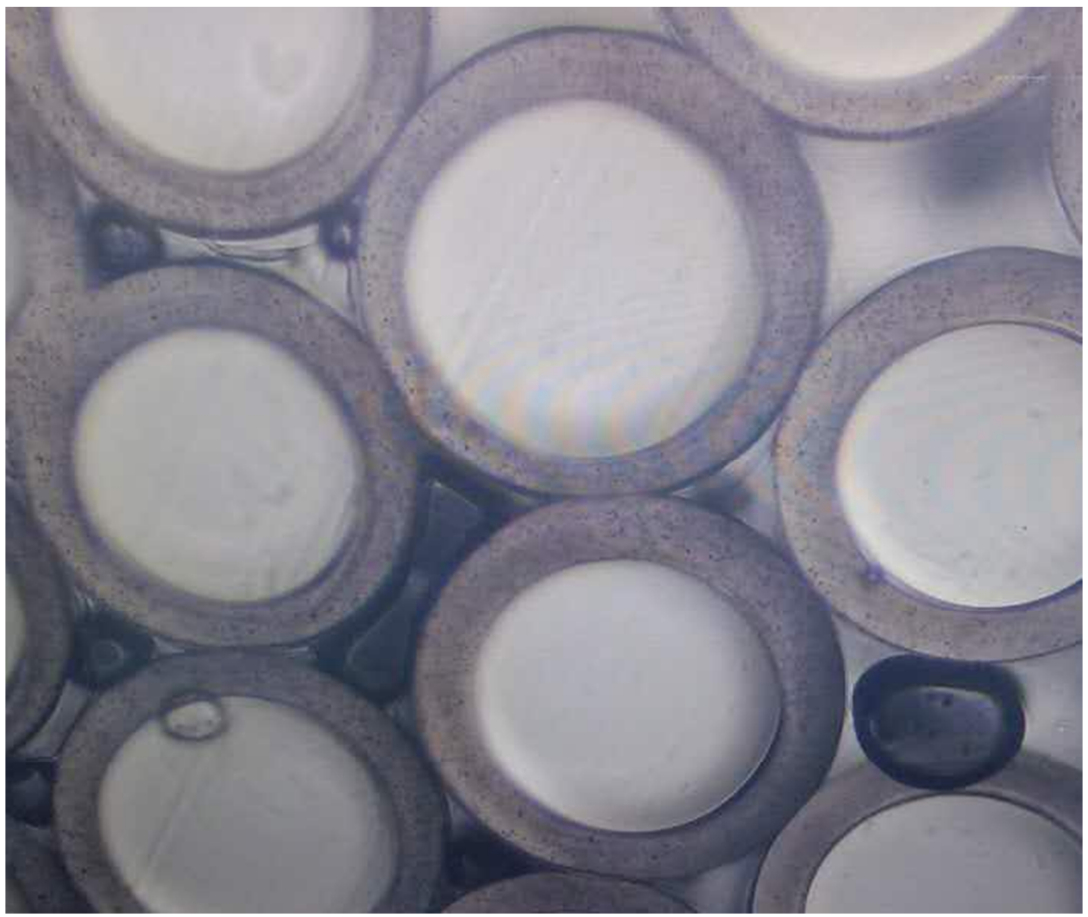

Figure 1. Primary fiber cross section. 
Using Bio-based polyamides 56 as the cortical layer structures of composite fiber, we can improve the wearing comfort and dyeing effect of fabrics and enhance the soft and smooth feeling of fiber because PA56 is easy to be dyed and has a good flexibility. Due to the low density of polyamide 56, we can reduce the weight of the fabric. The finished fabrics have light and wear-resisting effect. Because the PET rigidity is larger, it can keep conformity of the fabric by using PET as the core layer of the composite fiber. Because it is not easy to absorb water So it has washable and quick-drying properties. This sheath-core composite fiber fabric has better moisture absorption and perspiration, antistatic and feels light and soft. The composite proportion in 20: 80-50: 50 was relatively appropriate. Experiments show when the compound ratio is at 30: 70 the fiber moisture absorption perspiration and the comprehensive performance of the optimal is best.

Because the raw material of Bio-based polyamides 56 is produced by fermentation. It often contains more groups with pigment and the fiber is yellow. It will affect the color and luster of fabric. Especially it is high-end fabrics is difficult to meet the requirements of high-end fabrics. It is a good way to optimize the fiber color values that adding a suitable amount of modified fluorescent whitening agent in the spinning process. Experiments show that the color and luster of fiber can reach to the state of normal product by adding fluorescent whitening agent at $0.4-0.5 \%$ to the Bio-based polyamides 56 .

\subsection{Chip Drying}

Bio-based polyamides 56 hygroscopicity is very strong and its melting point is high. If high temperature is used in the process of spinning it is easy to hydrolysised by heat. As the same time the pet is also easier to occur hydrolysis by heat ${ }^{[3]}$ the condition of drying is higher and the crystallization and drying time is longer than the commonly product. Adopting low temperature and-long time drying methods, we can ease the thermal degradation of fiber macromolecule and avoid the melt viscosity and finished product strength to occur greatly changes and fluctuations [3]. At the same time if the water content is too high, it will aggravate the hydrolysis of macromolecular chain segment and it is easy gasification to form bubbles or will make shear rate and elastic factor increases. Then non-Newtonian index rise. In the high shear rate and high extrusion pressure of high speed spinning, the melt extruding effect increase. It will cause the melt spin ability broken, the fiber forming hard $^{[1]}$ and product yellow. Experimental results show: bio-based polyamides 56 crystallization temperature 135-145. C, time at 25 to $35 \mathrm{~min}$; drying temperature 130-145. C, drying time 25-35 h, PET chip crystallization temperature 155-155. C, time of 30-40 min; drying temperature 153-160. C, drying time 8-11 h,; fluorescent brightener drying temperature 115-125. C, the drying time $5-8 \mathrm{~h}$, the effect is good. All the ingredients viscosity drop is less than $0.0023 \mathrm{dl} / \mathrm{g}$ and the moisture content is about 21PPM. It has a good spin ability.

\subsection{Spinning Process}

Because the melting point of Bio-based polyamides 56 is close to PET, So the spinning temperature process is very similar. But in order to alleviate the yellowing of Bio-based polyamide 56, the spinning temperature of Bio-based polyamides 56 is low. In order to ensure the chip feed smoothly, prevent the chip in front of the screw premature melted and prevent the melt flowing back the temperature of the ahead zone of the extruder must is low. In order to ensure the product strength and toughness and ensure that the yarn without oil viscosity is less than $0.0025 \mathrm{dl} / \mathrm{g}$. But if the spinning temperature is too low to ensure the chip melt fully it will affect the rheological properties of the melt and effect the fiber forming ${ }^{[3]}$. Experimental results show: PET spinning biphenyl gas temperature control in 290-298. C range, the after-filter pressure $92-96 \mathrm{mpa}$, polyamide 56 spinning biphenyl gas temperature control in 280-288. C range, the after-filter pressure 90-95 mpa, fluorescent whitening master batch content $4.2 \%$ (The active ingredients of the master batch is $10 \%$ ), it has the extremely good spin ability and product style.

Because the polyamide 56 has good flexibility, the melt Will expand to a large degree. In order to keep the uniformity of the sheath-core composite structure and fiber quality, the spinning speed is a bit low. But if the spinning speed is too low, not only the production cost is too high, but also the strength of fiber will be declined. In general, the POY spinning speed in the rage of 2800-3000, FDY spinning speed in 4000-4300 $\mathrm{m} / \mathrm{min}$ is more appropriate.

Bio-based polyamides 56 belongs to semi-crystalline polymer, crystallization rate and crystallinity were relatively high. In order to improve the macromolecule relaxation degree of polyamide 56 , reduce the skin-core structure of polyamide 56 caused by the difference between inside and outside the skin-core structure, cooling condition must be ease. So it can avoid that the polyamide 56 at the cortex part was cracked or breakage during tensile deformation processing composite fiber in the machining process during tensile deformation processing. Thus we can keep the product dyeing effect and use performance. But if the cooling conditions of melt is excessively mitigatory, the melt form into fiber too slowly and monofilament of fiber is easy adhesion together. This will affect processing and performance. Experiments show that side wind temperature 23 to 28 . C; wind speed at $0.40-0.50$ $\mathrm{m} / \mathrm{s}$; Rheumatism is $80-90 \%$, the product has good skin-core structure and after-processing and using performance.

In order to ensure the combine fastness of two-component of sheath-core composite structure and keep the smoothness of cortex structure, the drawing process is relatively slow, and draw ratio is relatively low. If drawing speed is too fast, drawing ratio is too large, the fiber sheath layer structure is easy to crack, uneven dyeing easily, occurring streaks, even monofilament rupture, and easy to form the fuzziness. But if the draw speed is too slow, it can reduce production speed too big. The cost of production will increase. It will decrease the 
product benefits. If the Draw ratio is too low, the fiber will have frozen point or frozen yarn. It will make the product quality and performance bad. Experimental results show that the draw ratio of FDY at 2.5- 3.0 is relatively appropriate. If the spinning speed of POY is $2800-3000 \mathrm{~m} / \mathrm{min}$ or so, we can keep the suitable spinneret drawing ratio and fiber drawing speed then the finished fiber has good composite structure and good processing performance.

\subsection{The False Twist Texturing Process}

The processing speed of bio-based polyamides 56 sheath-core composite fiber should be slightly lower. Because the false twist drawing texturing speed is slow, so the false twist drawing texturing temperature must be lower. we can choose it according to the requirement for crimp elasticity. If we closed the second heat box, the fiber elasticity is best. If the production speed is too high, the drawing speed is too fast, the temperature is too high, it can make the yarn running instability, jittering in the twist texturing. The finished fiber will easy to occur the fuzziness or frozen point or frozen yarn. But if the speed is too slow, the cost of production will increase. If the temperature is too low, the heat that twist texturing need is not enough. It will influence the false twist texturing effect and the product fluffy performance and elastic turn bad. Especially the temperature of the second heat box will greatly affect the finished product fleeciness. We would design the temperature in accordance with the requirements of us to enhance flexibility and fluffy performance and the second heat box can be closed. In a general, the yarn speed: 620-680, draw ratio: $1.65-1.70, \mathrm{D} / \mathrm{Y}: 1.520$ to 1.580 , the first heat box temperature: $185-192$. C, and the second heat box temperature: $150-160$ or close, OF2: $5.006 .00 \%$, OF3: 4.60 to $5.20 \%$

Because the bio-based 56 sheath-core composite modified polyamide fiber has certain elasticity, the winding tension should be not too high. If the winding tension is too high, it is easy molding or occurring spider silk. But if the winding tension is too low, the product is easy to collapse and deformation in the packing and shipping and affect the unwinding performance. The winding Angle should increases appropriate to improve the phenomenon such as convex. But if the winding Angle is too big, It is easy to produce spider silk. The Spinning oil demand having good smoothness, oil film strength, and have good permeability, to ensure that the oil uniformity and protect the yarn from damaging of surface friction. So as to ensure the fiber has good using performance [4]. Experimental results show: using Japanese Takemoto oil Co., Ltd PET N - 2087 FDY oil and oiling in the rage of 1.0-1.2\%, using Japanese Takemoto oil Co., Ltd F - 582 POY oil and oiling in $0.5-0.8 \%$, The fiber has good processing properties and using performance.

\section{Conclusion}

1) Using The bio-based polyamides 56 and polyester as the raw material, adopting sheath-core composite mode of production, using the polyamide 56 as the sheath layer structure and adding a certain proportion fluorescent whitening agent in it, controlling the production technology of spinning and false twist texturing process well, we can produce bio-based polyamides 56 sheath-core composite modified fiber with good quality color, hygroscopic, antistatic and resilient properties, good wearing comfort, soft and smooth feel.

2) By the sheath-core composite production technology of bio-based polyamides 56 and PET, we can improvement the properties of PET products and effectively expand the application field and development space of bio-based polyamides 56 and PET.

\section{References}

[1] Weicai Yu. The analysis on Physical properties and spinnability of nylon 56 [J]: Polyester Industr, 2014(1), p. 38-39.

[2] Jinyu Wang, Huaping Wang, Bimei Chen. The research on shrinkage behavior of Polyester polyamide composite fiber [J]: Synthetic fiber industry, 2002, 25(10): 18-21.

[3] Weicai Yu. Physical properties and spinnability analysis of nylon 56 [J]: Polyester industry, 2014(1): 38-39.

[4] Haifeng Yu, Meigui Yang. Discussion on PET/PTT compound fiber technology [J]: Synthetic Fiber, 2011, 34 (6): 53-55.

[5] Morales-Gámez, L., D. Soto, L. Franco, and J. Puiggalí, Brill transition and melt crystallization of nylon 56: An odd-even polyamide with two hydrogen-bonding directions, Polymer, 2010, 51, (24), p. 5788-5798.

[6] J. R. Araujo, C. B. Adamo, M. V. Costa e Silva, M.-A. De Paoli. Antistat ic-Reinforced Bioco mposites of Polyamide- 6 and Polyaniline- Coated Curaua Fibers Prepared on a Pilot Plant Scale [J]. polymer compsites, 2013, 10(1002), p. 1081-1090.

[7] Huang X, Li C, Guan G, et al. Crystallization kinetics, melting behavior, And morphologiesofpoly (butylenesuccinate) andpoly (butylenesuccinate)-block-poly (propylene glycol) segmented copolyester [J]. Journalof Applied polymer Science, 2010, 118(4): 2225-2235.

[8] Samanta, S., J. He, S. Selvakumar, J. Lattimer, C. Ulven, M. Sibi, J. Bahr, and B. J. Chisholm, Polyamides based on the renewable monomer, 1,13-tridecane diamine II: Synthesis and characterization of nylon 13,6, Polymer, 2013, 54, (3), p. 1141-1149.

[9] Study on preparation and performance of Nylon $6 /$ nano montmorillonite composite material [J]. Synthetic fiber, 2013, 42(3), p. 1-6.

[10] Yuan Zong. Preparation and Properties of Nano composite metal oxides/polyamide composite fiber [J]. Synthetic fiber, 2013, 42(3), p. 1-6.

[11] Magill, J. H. Spherulitic Crystallization. Part I. "Odd-Even" Polyamides: Nylon 56 and Nylon 96, Journal of Polymer Science: Part A Vol. 965, 3, p. 1195-1219.

[12] Berda E B, Foster E J, Meijer E W. Toward controlling folding in synthetic polymers: Fabricating and characterizing supramolecular single-chain nanoparticles [J]. Macromolecules, 2010, 43, p. 1430-1437. 